\title{
Anomalía de Ebstein y aneurisma del seno coronario
}

\author{
Ebstein's anomaly and coronary sinus aneurysm \\ William F. Bautista-Vargas* \\ Servicio de Electrofisiología Cardiovascular, Hospital Departamental Santa Sofía, Manizales, Colombia
}

\section{Resumen}

La anomalía de Ebstein es una cardiopatía congénita poco común que se asocia a la presencia de vías de conducción anómalas y episodios de taquicardia supraventricular frecuentes, algunos inestables. La asociación con alteraciones anatómicas del seno coronario es rara y no ha sido reportada. Se presenta el caso de una paciente de 58 años con enfermedad coronaria, anomalía de Ebstein, episodios de taquicardia ortodrómica y aneurisma del seno coronario, a quien se realizó ablación.

Palabras clave: Anomalía de Ebstein. Vía accesoria. Aneurisma del seno coronario.

\begin{abstract}
Ebstein's disease is a congenital cardiomyopathy, with a low prevalence in the general population. This abnormality has been associated with abnormal cardiac conduction problems, one of the most important being the accessory pathways. In the presence of an accessory pathway, frequent supraventricular tachycardias may occur, some of which are poorly tolerated. The association with the anomalies of the coronary sinus is not currently reported. The case of a 58-year-old woman with Ebstein's disease, episodes of supraventricular tachycardia, and coronary sinus aneurysm undergoing ablation therapy is presented.
\end{abstract}

Key words: Ebstein's anomaly. Abnormal accessory pathway. Coronary sinus aneurism.

\section{Introducción}

La anomalía de Ebstein fue descrita por primera vez por Wilhelm Ebstein en 1886 en un estudio anatomopatológico de un paciente de 19 años. Esta cardiopatía se presenta en el $1 \%$ de la población, representa el $40 \%$ de las cardiopatías congénitas con compromiso de la válvula tricúspide ${ }^{1}$ y tiene una prevalencia de 5.2 por cada 100,000 nacidos vivos ${ }^{2}$. Se caracteriza por seis hallazgos principales: falla en la formación de las valvas de la válvula tricúspide, desplazamiento apical de la válvula tricúspide con insuficiencia, fenestraciones y movimiento anormal de la valva anterior, dilatación del corazón derecho, comunicación interauricular hasta en el $75 \%$ de los casos, fibrilación auricular y arritmias. El $25 \%$ de los pacientes presentan vías accesorias relacionadas ${ }^{2,3}$.

Existen cardiopatías congénitas asociadas a la anomalía de Ebstein, entre las cuales están los defectos del tabique interauricular $(80 \%)$, seguidos de estenosis o atresia pulmonar $(30 \%)$, comunicación interventricular $(4.3 \%)$, coartación de aorta y tetralogía de Fallot $^{1,2}$; sin embargo, las malformaciones conjuntas

\section{Correspondencia:}

*William F. Bautista-Vargas

E-mail: williambautista679@icloud.com
Disponible en internet: 10-06-2021 Rev Colomb Cardiol. 2021;28(2):180-184 www.rccardiologia.com 0120-5633 / C 2020 Sociedad Colombiana de Cardiología y Cirugía Cardiovascular. Publicado por Permanyer. Este es un artículo open access bajo la licencia CC BY-NC-ND (http://creativecommons.org/licenses/by-nc-nd/4.0/). 
del seno coronario no han sido reportadas en la literatura.

La sintomatología en los adultos se caracteriza por la presencia de arritmias auriculares recurrentes y refractarias al tratamiento farmacológico, además de cianosis progresiva e incremento de la disnea ${ }^{1}$.

Existe una amplia variedad de arritmias reportadas en relación con la anomalía de Ebstein, entre las cuales se encuentran taquicardia por reentrada auriculoventricular, flutter auricular, fibrilación auricular, taquicardia de reentrada intranodal, taquicardia ventricular monomórfica y taquicardia ventricular polimórfica, que tal vez se deban a la alteración de la hemodinamia del ventrículo derecho ${ }^{4}$.

La clasificación más aceptada de la anomalía de Ebstein es la de Carpentier, que la divide en cuatro categorías: tipo $A$, caracterizada por ventrículo derecho funcional con volumen normal; tipo $B$, en la que la parte atrializada del ventrículo derecho es larga, pero la valva anterior se mueve libremente; tipo $\mathrm{C}$, con restricción importante del movimiento de la valva anterior y obstrucción del tracto de salida del ventrículo derecho; y tipo $D$, con el ventrículo derecho gravemente atrializado, a excepción de una pequeña porción infundibular².

El estudio electrofisiológico y la ablación percutánea se han propuesto como intervenciones seguras y eficaces para el tratamiento de los pacientes sintomáticos.

\section{Caso clínico}

Mujer de 58 años con antecedentes de anomalía de Ebstein tipo $\mathrm{C}$ no corregida, ablación de dos vías de conducción anómala extrainstitucionales hace más de 1 año, reportadas en las regiones posteroseptal y anteroseptal derechas; además, historia de enfermedad coronaria e implante de tres stents (dos en las arterias coronarias diagonales y un stent medicado en la coronaria derecha en el último mes). Consultó por un episodio de palpitaciones precordiales de comportamiento incesante, inicio súbito e inestabilidad hemodinámica; requirió tratamiento con cardioversión eléctrica. Una vez realizada la intervención, y sin encontrar evidencia de preexcitación manifiesta en el electrocardiograma, se admitió para realizar el estudio electrofisiológico con mapeo tridimensional y ablación de la taquicardia supraventricular.

El procedimiento se realizó bajo sedación consiente. Se colocaron accesos vasculares femorales bilaterales y luego se incidió con catéteres diagnósticos duodecapolar (crista-seno) y cuadripolares a las regiones His y ápex del ventrículo derecho, con lo cual se pudo efectuar el estudio electrofisiológico. En este se documentó taquicardia ortodrómica secundaria a una vía de conducción anómala oculta posteroseptal. Se realizó reconstrucción anatómica y visualización con ecografía intracardiaca (Fig. 1), e inyección de contraste en el seno coronario, con evidencia de retardo de llenado y presencia de un aneurisma fusiforme del seno coronario (Fig. 2) (Vídeo 1).

Durante el estudio electrofisiológico se determinaron:

- Intervalos intracavitarios en ritmo sinusal AH: $60 \mathrm{~ms}$ HV: 61 ms (Fig. 1).

- Inducción de taquicardia con estimulación desde el ápex del ventrículo derecho (600-350 ms) y primoactivación a nivel del seno coronario 7-8:

- La taquicardia con despolarización excéntrica con longitud de ciclo de $446 \mathrm{~ms}$, VA $317 \mathrm{~ms}$.

- Intervalo de retorno: $599 \mathrm{~ms}$, delta de AH: $38 \mathrm{~ms}$, PPITCL (delta de AH): $94 \mathrm{~ms}$, compatible con taquicardia ortodrómica secundaria a vía de conducción anómala oculta, por medio de entrainment de la taquicardia con sobreestimulación ventricular continua (Fig. 1).

Se realizó el mapa de activación en taquicardia con el sistema de mapeo tridimensional Ensite (St. Jude Medical, St. Paul, Mn, USA). La actividad auricular fue más precoz en el piso del seno coronario; posteriormente, se aplicaron puntos de ablación (20 Wattz y $42{ }^{\circ} \mathrm{C}$ con catéter bidireccional irrigado) durante la taquicardia, lo que dio como resultado final el cese de la arritmia (Fig. 2).

Durante la comprobación posterior a los 30 minutos de espera y con dobutamina a dosis de $20 \mu \mathrm{g} / \mathrm{kg}$ por minuto no se indujo ningún tipo de arritmia con estimulación auricular y ventricular.

En el área de recuperación, la paciente desarrolló hipotensión sostenida, la cual respondió adecuadamente a la administración de líquidos intravenosos, y no se observó evidencia de derrame pericárdico; lo anterior se consideró secundario al efecto de la sedación.

Durante el seguimiento a 3 meses no se detectó ningún tipo de recurrencia arrítmica.

\section{Discusión}

La ablación de la taquicardia supraventricular es un procedimiento desafiante en pacientes con cardiopatías congénitas; usualmente, este tipo de pacientes requieren uno o más intentos de ablación ${ }^{5}$.

Se presenta el caso de una paciente de 58 años con síntomas secundarios a anomalía de Ebstein tipo C, 


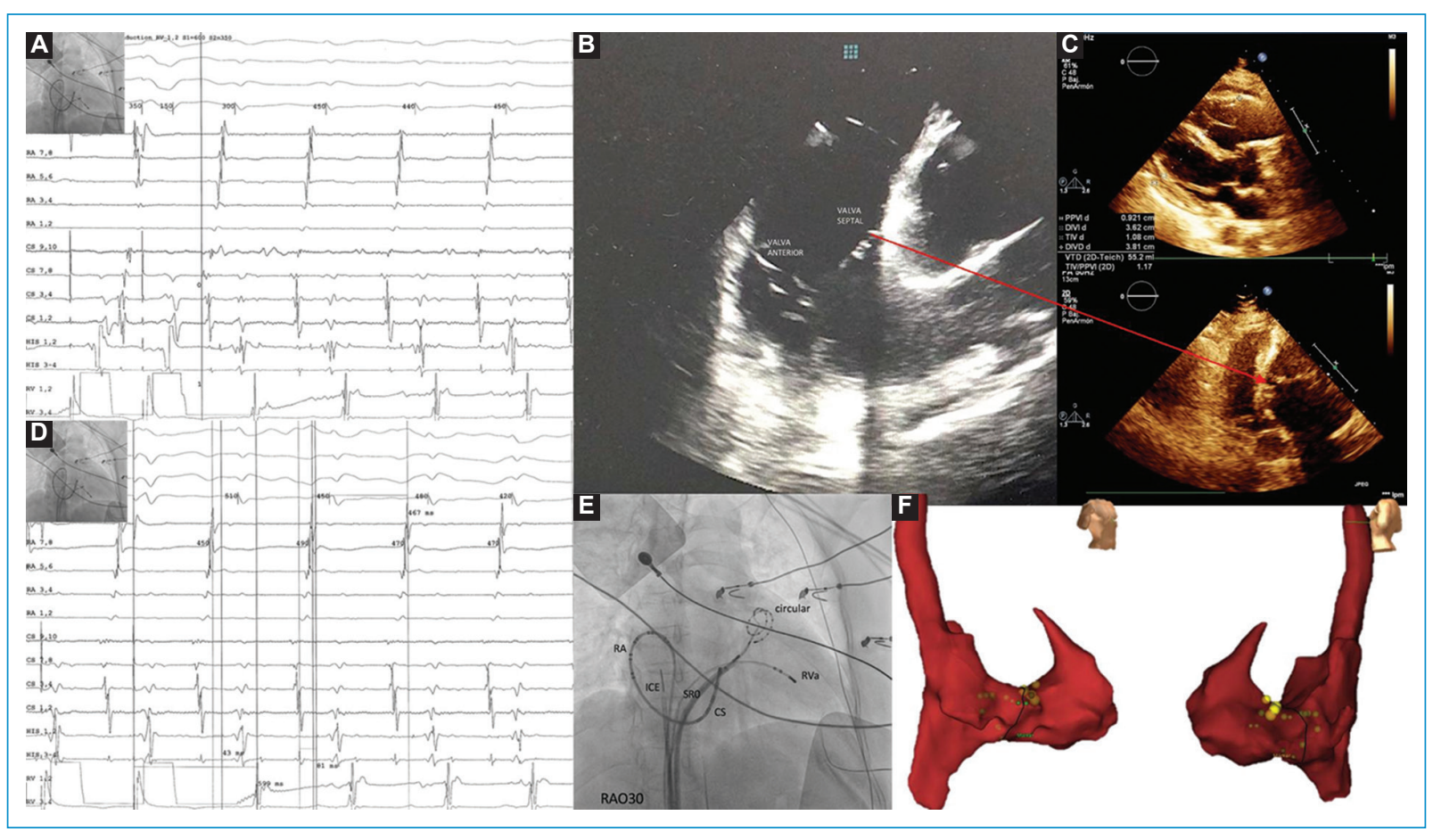

Figura 1. A: inducción de taquicardia con estimulación ventricular. B: imagen de ecografía intracardiaca desde Home View que muestra la valva septal y la valva anterior de la tricúspide, así como parte del tracto de salida del ventrículo derecho. C: vista paraesternal del ecocardiograma transtorácico y vista en el eje largo de cuatro cámaras en la que se aprecia la inserción baja de la valva septal y anterior. D: entraiment con estimulación ventricular con intervalo de retorno y mediciones. E: vista en oblicua derecha que muestra el catéter circular, ecografía intracardiaca (ICE), catéter crista seno, His, ápex del ventrículo derecho y catéter circular. F: mapa anatómico realizado con el catéter circular. CS: seno coronario; ICE: eco intra-cardiaco; RA: aurícula derecha; RA030: proyección oblicua anterior derecha 30 grados; RVa: apex del ventricle derecho; SRO: camisa no deflectable.

además de enfermedad coronaria, sin síntomas de falla cardiaca. En el electrocardiograma de base se halló un bloqueo de rama derecha, concordante con lo reportado por Iturralde et al. ${ }^{5}$; en pacientes con anomalía de Ebstein, taquicardia supraventricular y ausencia de bloqueo de rama derecha es común encontrar una vía de conducción anómala en el estudio electrofisiológico, con una sensibilidad del $98 \%$, una especificidad del $92 \%$ y unos valores predictivos positivo y negativo del $91 \%$.

En el estudio electrofisiológico se diagnosticó una vía de conducción anómala oculta, con evidencia de taquicardia ortodrómica. En la literatura se ha reportado la presencia de vías anómalas de conducción con Wolff-Parkinson-White en un $30 \%$ a $56 \%$ de los casos $^{1-3}$; estas son múltiples hasta en el $29 \%$, de pared lateral posterior del ventrículo derecho en el $62 \%$, septal derecha en el $34 \%$, posteroseptal en el $21 \%$, anteroseptal en el $5 \%$, de ventrículo izquierdo hasta en el $4 \%$, e incluso con carácter decremental tipo Mahaim en un $3 \%$; las vías de conducción anómalas sin preexcitación u ocultas se presentan hasta en el $9 \%$ de los casos ${ }^{2,5-7}$.
La paciente del caso tenía antecedente de ablación extrainstitucional de dos vías de conducción anómala con persistencia de palpitaciones, además de un electrocardiograma en el que se documentó taquicardia supraventricular de carácter inestable, la cual tuvo que ser cardiovertida eléctricamente para su tratamiento; luego, fue llevada a un segundo procedimiento en el cual se logró el tratamiento definitivo de la arritmia.

En cuanto a la frecuencia de éxito de la terapia con ablación en pacientes con anomalía de Ebstein, se describen porcentajes del $100 \%$ para taquicardia de reentrada intranodal, del $77 \%$ para taquicardia ortrodrómica y del $50 \%$ para flutter auricular ${ }^{8}$. El porcentaje de recurrencia de la taquicardia auriculoventricular posterior a la terapia de ablación es de un $40 \%$; se han reconocido ciertas circunstancias que podrían explicar este hecho, como la baja amplitud de los electrocardiogramas durante el mapeo de la continuidad auriculoventricular, el recorrido oblicuo y la orientación de las vías de conducción anómalas, la presencia de múltiples vías de conducción anómalas, la dilatación de las 


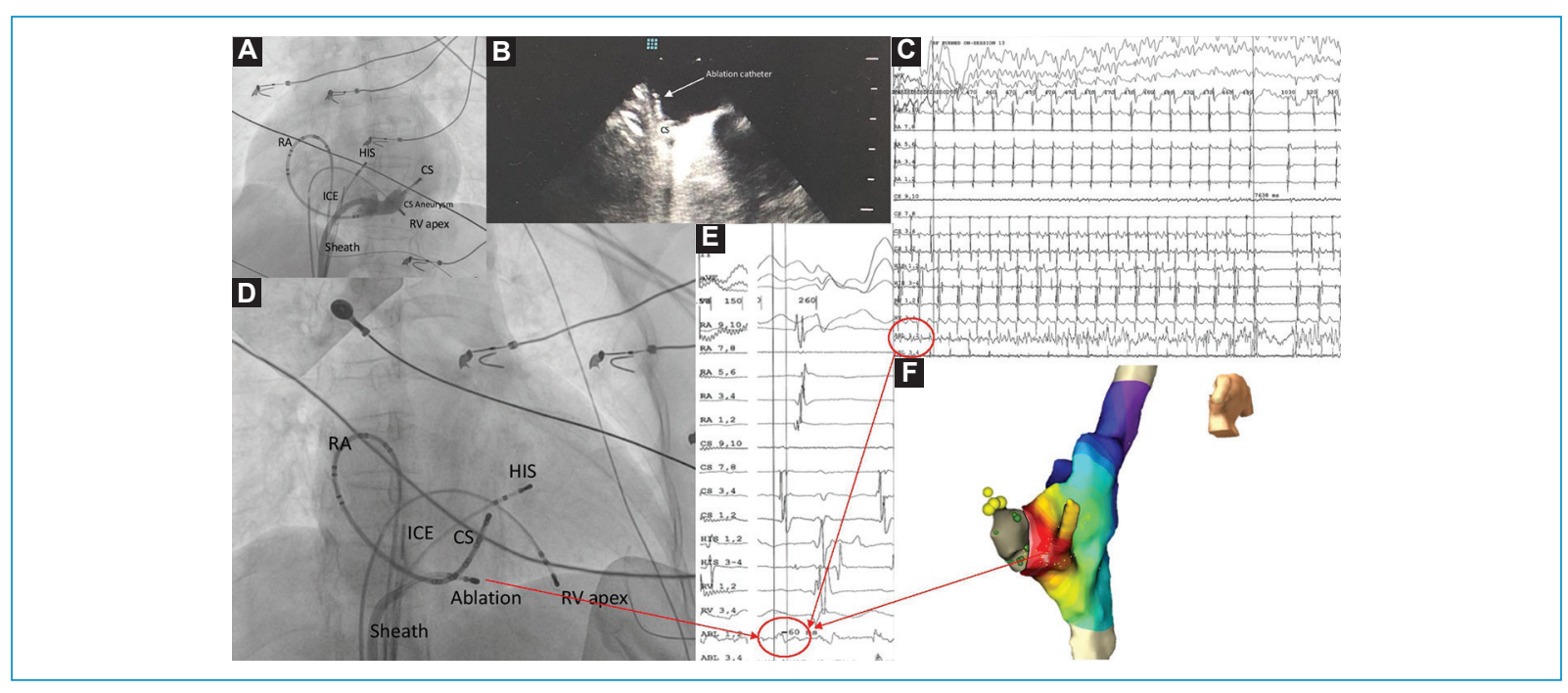

Figura 2. A: imagen en oblicua izquierda que muestra la inyección de contraste en el seno coronario, con evidencia de un aneurisma fusiforme del seno. B: ecografía intracardiaca que muestra el catéter de ablación en el piso del seno coronario. C: ablación con cese de la arritmia a los 7 segundos de aplicación de radiofrecuencia. D: imagen en oblicua derecha que muestra el catéter de ablación en el piso del seno coronario. E: electrocardiograma que muestra precocidad de -60 ms respecto a la onda P. F: mapa de activación que muestra el sitio de mayor precocidad. ABL: Electrograma de ablación; HIS: posición del HIS- no es una sigla. ICE: Ecografia Intra-cardiaca; RA: auricle derecha; $\mathrm{RV}$ : ventricle derecho; RVapex:Apex del ventricle derecho.

cavidades derechas, la ausencia de un verdadero anillo tricuspídeo anatómico y la intolerancia hemodinámica para el mapeo electroanatómico durante la taquicardia $^{4}$. Otra alternativa a la ablación percutánea es la ablación «a cielo abierto» (que se realiza durante la cirugía cardiaca), con plastia o cambio de la válvula tricúspide, en la cual se han reportado buenos resultados a mediano plazo y una mortalidad del $4.8 \%{ }^{6}$.

En la ablación realizada a esta paciente se utilizó ecografía intracardiaca, la cual permitió observar mejor la anatomía tricuspídea, asegurar el contacto con el catéter durante la reconstrucción anatómica, verificar el contacto durante la ablación por radiofrecuencia y asegurarse de la ausencia de complicaciones al final del procedimiento. El uso de ecocardiografía intracardíaca, así como el de sistemas de mapeo tridimensional y de catéteres de mapeo intracoronario, se ha propuesto como estrategia para aumentar el porcentaje de éxito del procedimiento ${ }^{8,9}$.

Dado que en este caso se encontró que el seno coronario fue el sitio de máxima precocidad auricular, se procedió a realizar una inyección de medio de contraste, con lo cual se evidenció estasis y deformación fusiforme que configuraban un aneurisma. En la literatura no se ha encontrado asociación de anomalía de Ebstein y presencia de aneurisma del seno coronario, y por lo tanto se considera importante documentar este caso.

\section{Conclusiones}

La ablación de la taquicardia supraventricular en los pacientes con anomalía de Ebstein es un desafío para el electrofisiólogo. La ausencia de bloqueo de rama derecha en los pacientes con anomalía de Ebstein y taquicardia es altamente sugestiva de una vía de conducción anómala que participe en la taquicardia. El uso de ecografía intracardiaca permite la toma fidedigna de puntos para la elaboración de mapas tridimensionales, establecer la anatomía en función del procedimiento de ablación y asegurar un contacto efectivo durante las aplicaciones de radiofrecuencia.

Las cardiopatías congénitas hacen más probables los hallazgos anormales durante los procedimientos de electrofisiología.

\section{Conflicto de intereses}

De forma libre y abierta manifiesto que no existe conflicto de intereses para la presentación de este caso.

\section{Responsabilidades éticas}

Protección de personas y animales. Los autores declaran que para esta investigación no se han realizado experimentos en seres humanos ni en animales. 
Confidencialidad de los datos. Los autores declaran que han seguido los protocolos de su centro de trabajo sobre la publicación de datos de pacientes.

Derecho a la privacidad y consentimiento informado. Los autores han obtenido el consentimiento informado de los pacientes y/o sujetos referidos en el artículo. Este documento obra en poder del autor de correspondencia.

\section{Bibliografía}

1. Booker OJ, Nanda NC. Echocardiographic assessment of Ebstein's anomaly. Echocardiography, 2015;32 (Suppl 2):S177-88.

2. Negoi RI, Ispas AT, Ghiorghiu I, Filipoiu F, Negoi I, Hostiuc M, et al. Complex Ebstein's malformation: defining preoperative cardiac anatomy and function. J Card Surg. 2013;28:70-81.
3. Wei W, Zhan X, Xue Y, Fang X, Liao H, Deng $\mathrm{H}$, et al. Features of accessory pathways in adult Ebstein's anomaly. Europace. 2014;16:1619-25.

4. Walsh EP. Ebstein's anomaly of the tricuspid valve: a natural laboratory for re-entrant tachycardias. JACC Clin Electrophysiol. 2018;4:1271-88. Disponible en: https://doi.org/10.1016/j.jacep.2018.05.024.

5. Iturralde $P$, Nava S, Sálica G, Medeiros A, Márquez MF, Colin L, et al Electrocardiographic characteristics of patients with Ebstein's anomaly before and after ablation of an accessory atrioventricular pathway. J Cardiovasc Electrophysiol. 2006;17:1332-6.

6. Khositseth A, Danielson GK, Dearani JA, Munger TM, Porter CJ. Supraventricular tachyarrhythmias in Ebstein anomaly: management and outcome. J Thorac Cardiovasc Surg. 2004;128:826-33.

7. Rodríguez LR, Iturralde P, Calderón-Colmenero J, Buendía A. Ablación transquirúrgica de vía accesoria atrioventricular en anomalía de Ebstein. Arch Cardiol Mex. 2005;75:421-4

8. Orczykowski M, Derejko P, Bodalski R, Urbanek P, Zakrzewska-Koperska J, Sierpiński R, et al. Radiofrequency catheter ablation of accessory pathways in patients with Ebstein's anomaly: at 8 years of follow-up. Cardiol J. 2017;24:1-8.

9. Vukmirović M, Peichl P, Kautzner J. Catheter ablation of multiple accessory pathways in Ebstein anomaly guided by intracardiac echocardiography. Europace. 2016;18:339. 